\title{
Characterization of red fluorescent reporters for dual-color in vivo three-photon microscopy
}

\author{
Michael A. Thornton ${ }^{1,2}$, Gregory L. Futia ${ }^{3}$, Michael E. Stockton ${ }^{1,2}$, Baris N. Ozbay ${ }^{4}$, Karl Kilborn ${ }^{4}$, Diego \\ Restrepo $^{1,2}$, Emily A. Gibson ${ }^{2,3,5}$, Ethan G. Hughes ${ }^{1,2,5}$
}

Three-photon (3P) microscopy significantly increases the depth and resolution of in vivo imaging due to decreased scattering and nonlinear optical sectioning. Past studies used separate 1300 and $1700 \mathrm{~nm}$ excitation sources to excite green and red fluorescent proteins. Recently work shows that a single 1300 $\mathrm{nm}$ excitation source allows for dual color 3P imaging with increased signal-to-background and decreased average power. Future use of single excitation 3P microscopes would reduce the need for additional custom optical elements and decrease cost. However, there is a lack of experimental data characterizing the excitation properties of specific fluorophore combinations in the near-infrared range. Here, we assess the dual-color imaging potential of tdTomato or mScarlet in combination with EGFP when excited by a single excitation source tuned from 1225-1360 nm in the living mouse brain. We find that tdTomato and $\mathrm{mScarlet}$, expressed in oligodendrocytes and neurons respectively, have exceptional signal-to-background in the 1300-1360 nm range in deep cortex, consistent with enhanced 3P cross sections. These results suggest that a single excitation source is advantageous for multiple applications of dual-color structural and functional brain imaging highlighting the importance of empirical characterization of individual fluorophores in the near-infrared region.

1. Introduction: Multiphoton microscopy methods allow for high-resolution structural and functional imaging deep into biological tissues ${ }^{1-3}$. Two-photon (2P) microscopy is a widely used technique to assess cellular and subcellular dynamics in the intact mouse brain. Although multiple excitation source $2 \mathrm{P}$ microscopy setups are increasingly common ${ }^{4,5}$, broad excitation profiles of specific green and red fluorescent proteins allow simultaneous dual-color $2 \mathrm{P}$ imaging using a single tunable excitation source ${ }^{6}$. This single-wavelength excitation dual-color $2 \mathrm{P}$ microscopy approach has benefited from careful characterizations of fluorescent protein excitation cross sections at relevant wavelength ${ }^{7-9}$ and enabled both functional and structural interrogation of neural dynamics in vivo. For example, simultaneous $1000 \mathrm{~nm}$ excitation of GCaMP6s / jRGECO1a permitted the detection of correlated axonal and dendritic calcium events in the visual cortex ${ }^{10}$, while $920 \mathrm{~nm}$ excitation of EGFP / tdTomato was used to track oligodendroglial differentiation and cell fate over time ${ }^{11}$. However, in vivo dual color $2 \mathrm{P}$ microscopy is practically limited in imaging depth to the superficial mouse cortex by light scattering and the generation of out-of-focus background.

The development of three-photon (3P) microscopy, which utilizes low rep-rate, high pulse energy excitation sources at $1300-1700 \mathrm{~nm}$, has significantly improved imaging depth and resolution due to decreased scattering and improved signal to background ratio ${ }^{3,12}$. 3P microscopy with $1700 \mathrm{~nm}$ excitation was first used in the intact mouse brain to image cortical and subcortical vasculature as well as RFP-labeled neurons throughout the visual cortex, cortical subplate, and hippocampus ${ }^{12}$. Since then, $1300 \mathrm{~nm}$ excitation of GCaMP6s has been used to record spontaneous neuronal activity throughout the cortical-subcortical volume ${ }^{13}$ and the sensitivity of these recordings and concomitant thermal and phototoxic changes have been characterized extensively ${ }^{14}$. Simultaneous dual color in vivo 3P microscopy remains challenging due to the large spectral separation in the optimal wavelength excitation windows for GFPs and RFPs, centered at 1300 and $1700 \mathrm{~nm}^{3,12}$, which currently necessitate independent dispersion compensation and custom optical elements with increased $1700 \mathrm{~nm}$ transmission.

Dual-color 3P microscopy of green and red fluorescent indicators was achieved in the fixed mouse brain using a novel two-stage optical parametric chirpedpulse amplifier seeded by a Ytterbium-doped fiber amplifier ${ }^{15}$ for $1300 \mathrm{~nm}$ and $1700 \mathrm{~nm}$ simultaneous output. However, issues associated with overlap of the two wavelengths at the focus and the requirement of higher powers $(\sim 1 \mu \mathrm{J})$ prohibit the application of this method to in vivo imaging in the mouse brain ${ }^{14,16}$. Alternatively, a recent report showed that single-wavelength $1300 \mathrm{~nm}$ excitation can be used with compatible combinations of fluorophores to achieve deep tissue simultaneous dual color imaging in the living mouse brain via higher-energy electronic excited states of the fluorophores ${ }^{17}$. However, a lack of information on the 3P excitation properties of commonly used fluorophores currently limits broad application of this approach. Here, we characterize the use of two RFPs with potentially enhanced excitation properties for in vivo single-source excitation 3P microscopy. These results will be important to future studies utilizing this method to perform cell fate tracking, cell localization for holographic stimulation, and subcellular neuronal and glial imaging deep in the living mouse brain.

\footnotetext{
${ }^{1}$ Department of Cell and Developmental Biology, University of Colorado School of Medicine, Aurora, CO, USA, ${ }^{2}$ Neuroscience Graduate Program, University of Colorado Denver, Anschutz Medical Campus, Aurora, CO, 80045, USA, ${ }^{3}$ Department of Bioengineering, University of Colorado Denver, Anschutz Medical Campus, Aurora, CO, 80045, USA, ${ }^{4}$ Intelligent Imaging Innovations (3i), Denver, CO, 80216, USA. ${ }^{5}$ Corresponding author. Email: emily.gibson@cuanschutz.edu; ethan.hughes@cuanschutz.edu
} 


\section{Methods:}

\subsection{Animals}

Animal experiments were conducted in accordance with protocols approved by the Animal Care and Use Committee at the University of Colorado Anschutz Medical Campus. Male and female mice used in these experiments were kept on a 14-h light-10-h dark schedule with ad libitum access to food and water and were housed with littermates. C57BL/6N MOBP-EGFP (MGI:4847238), Olig2tm1(cre/Esr1*)Htak (MGl:2183410), and B6.Cg-Gt(ROSA)26Sortm9(CAGtdTomato)Hze/J (JAX \#007909) were used for dual-color $3 \mathrm{P}$ imaging. Generation and genotyping of mice was performed as described previously ${ }^{11}$.

\subsection{Custom three-photon microscope}

A VIVO Multiphoton Open (3i) microscope, based on a Sutter Moveable Objective Microscope (MOM), was modified for three-photon imaging. The excitation source was a regenerative amplifier with $1030 \mathrm{~nm}$ center wavelength, $70 \mathrm{~W}$ average power, $<300 \mathrm{fs}$ pulse duration, adjustable repetition rate up to $2 \mathrm{MHz}$ (Spirit-1030-70, Spectra Physics), wavelength converted by a noncollinear optical parametric amplifier (NOPA-VIS-IR, Spectra Physics). The idler output of the NOPA is tunable in the $1200-2500 \mathrm{~nm}$ range. The laser was operated at a repetition rate of $1 \mathrm{MHz}$ and the final output power from the idler was $0.8-1.1 \mathrm{~W}$ at $1300 \mathrm{~nm}$. The power was modulated with a motorized half wave plate (KPRM1E/M - Ø1", Thorlabs). Beam conditioning of the NOPA output consisted of a Glan-Thompson prism, and expansion and collimating lens relay ( $\mathrm{f} 1=75 \mathrm{~mm}, \mathrm{f} 2=100 \mathrm{~mm}$, Newport), a $4 x$ reflective beam expansion telescope (BE04R, Thorlabs), and a beam demagnifying telescope $(\mathrm{f} 1=500$ $\mathrm{mm}, \mathrm{f} 2=200 \mathrm{~mm}$, Edmund Optics). The additional reflective beam expansion telescope and demagnification were to condition the beam size for a deformable mirror that was held flat for this study. Group delay dispersion (GDD) compensation was achieved using a prism compressor system consisting of two $25 \mathrm{~mm}$ SF10 prisms cut at Brewster's angle (10NSF10, Newport), and a gold roof mirror (HRS1015-M01, Thorlabs). The beam was directed to the galvanometers (Cambridge Technologies) and through a scan lens (Thorlabs SL50-3P), tube lens and a $760 \mathrm{~nm}$ long-pass primary dichroic. The back aperture of a high-NA multiphoton objective (XLPLN25XWMP2, 25X/1.05 NA, Olympus) was $\sim 75 \%$ filled for $3 \mathrm{P}$ imaging. The fluorescent emission was separated from the excitation path by the long pass dichroic mirror and spectrally filtered (green channel $=525 / 50 \mathrm{~nm}$, red channel $=620 / 60 \mathrm{~nm}$ ), and detected by photomultiplier tubes (H10770PA-40, Hamamatsu). Electronic signal was amplified, low-pass filtered, and digitized. Data were acquired with SlideBook 2021 (Intelligent Imaging Innovations).

\subsection{Two-photon microscopy}

2P images in Fig. S2 were generated using a wavelength tunable femtosecond oscillator (MaiTai-HP DeepSee,
Spectra Physics) combined with the 3-photon excitation path with a $1030 \mathrm{~nm}$ long pass filter after the prism compressor and aligned to co-propagate with the 1300 nm light.

\subsection{GDD Tuning and Pulse Measurements}

To tune the prism compressor for optimizing $3 \mathrm{P}$ excitation, we performed time lapse imaging of a green fluorescent flat slide and adjusted the prism separation and insertion in real-time to maximize the fluorescent signal. We checked but did not need to make any changes in the compressor for maximum signal over multiple months of imaging. Pulse measurements were made using frequency-resolved optical gating (FROG) (FROGscan, Mesa Photonics). The FROG has an allreflective optical path from its input to the $\mathrm{BBO}$ crystal used for second harmonic generation (SHG) to avoid adding any additional dispersion. For the same reason, a reflective objective 40x/.5 NA (LMM40X-UVV, ThorLabs) was used to collimate the beam after focusing through the objective, immersion water, and coverglass, followed by two silver mirrors to align into the FROG. This set up allowed us to characterize our pulse duration at the sample without additional dispersive elements that would change our pulse profile. We characterized the pulses for all wavelengths (Fig. 1, $1225-1360 \mathrm{~nm}$ ). For the 2P excitation, we used the internal GDD tuning on the MaiTai-HP DeepSee to find the maximum signal intensity at each wavelength.

\subsection{Stereotaxic AAV injections}

Six- to eight-week-old mice were anesthetized with Isoflurane inhalation (induction, 5\%; maintenance, 1.5$2.0 \%$, mixed with 0.5 liter per min $\mathrm{O}_{2}$ ) and kept at $37^{\circ} \mathrm{C}$ body temperature with a thermostat-controlled heating plate. A midline incision in the skin was made with fine surgical scissors to expose the skull. A small burr hole was made with a high-speed dental drill over the right forelimb primary motor cortex $(1 \mathrm{~mm}$ anterior and $1.5 \mathrm{~mm}$ lateral to Bregma) and the deep layer of the thinned skull was removed with a curved 27-gauge needle. To label layer V/VI cortical neurons, we made two 500nL injections of AAV8-hSyn-mScarlet-WPRE ${ }^{18}\left(1.5 \times 10^{12} \mathrm{vg} / \mathrm{mL}\right.$, Deisseroth lab, Stanford Viral Vector Core) at 1000 and $750 \mu \mathrm{m}$ depths below the pial surface at a rate of $100 \mathrm{~nL}$ / minute. The Injection needle was left in place for $5 \mathrm{~min}$. to prevent backflow and then removed slowly. The burr hole was filled with Vetbond (3M) and the skin incision was sutured using 5-0 Ethilon sutures.

\subsection{In vivo multiphoton microscopy}

Cranial windows were prepared as previously described ${ }^{19}$. Six- to ten-week-old mice were anesthetized and monitored as above. After removal of the skin over the right cerebral hemisphere, the skull was cleaned and a $2 \times 2 \mathrm{~mm}$ region of skull centered over either a) the posterior parietal cortex (-1 to $-3 \mathrm{~mm}$ posterior to bregma and 1 to $3 \mathrm{~mm}$ lateral), or b) the forelimb region of the primary motor cortex $(0-2 \mathrm{~mm}$ anterior to bregma and $0.5-2.5 \mathrm{~mm}$ lateral) was removed using a high-speed dental drill. A piece of cover glass (VWR, No. 1) was 
a

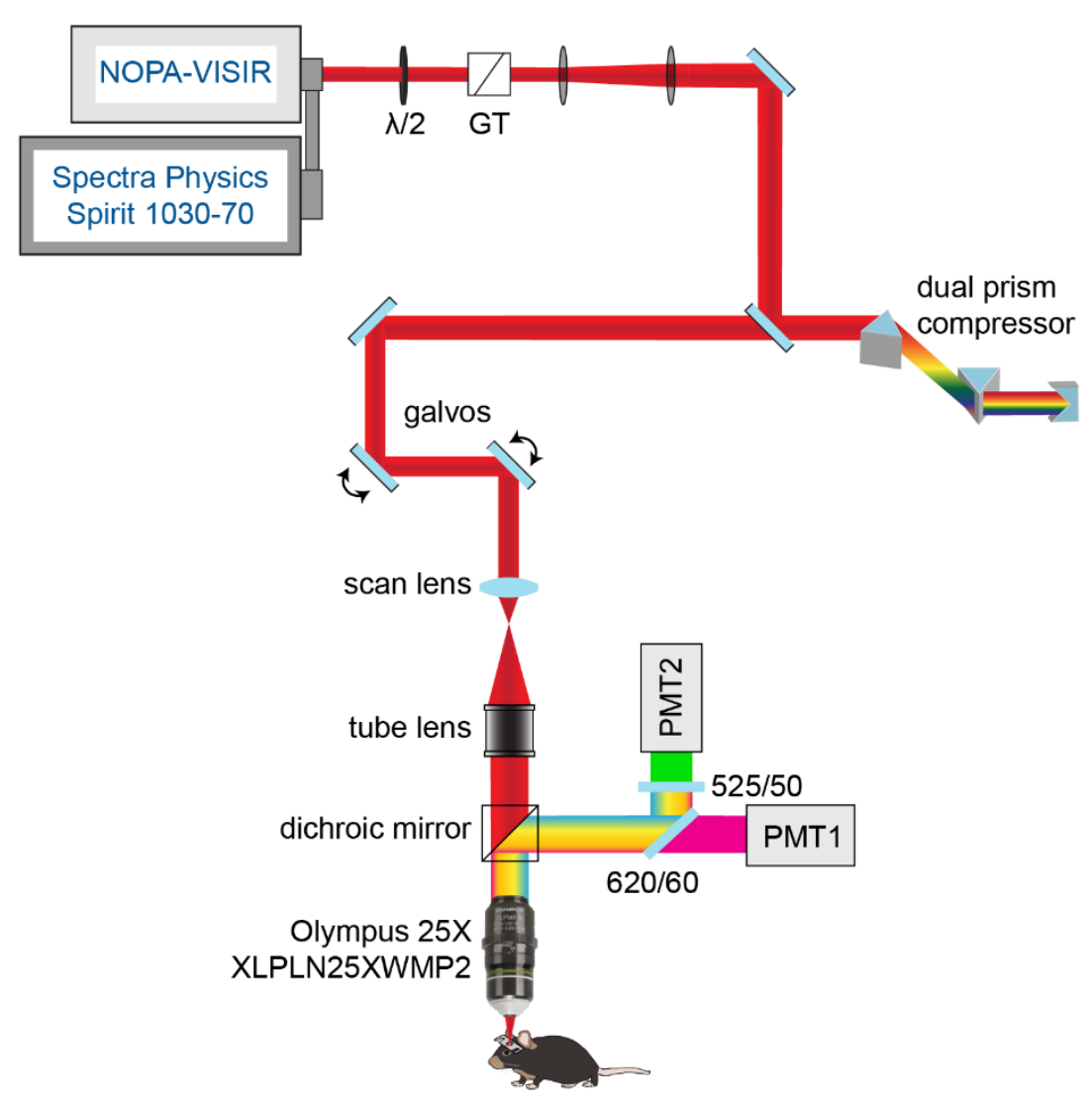

b

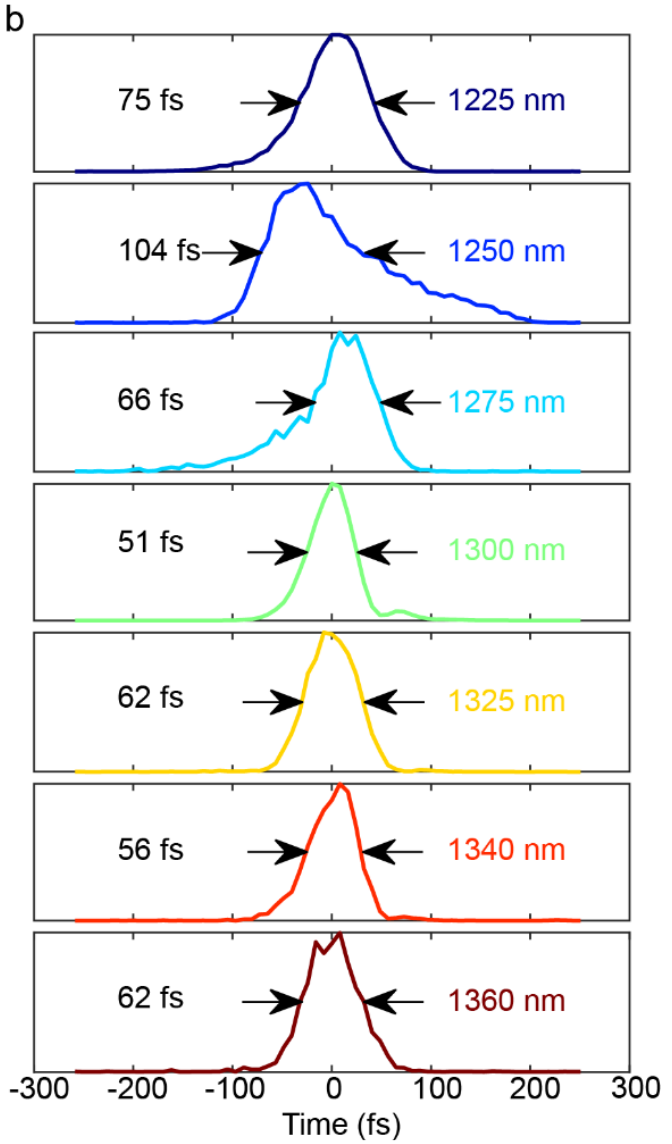

Figure 1: Custom three-photon microscope for dual color in vivo imaging. a) 3P light path and detection scheme for dual color imaging. b) Laser pulse temporal profile for wavelengths from $1200-1400 \mathrm{~nm}$, using frequency-resolved optical gating. Pulses were characterized at the objective focus. Pulse widths indicated are the full width at half maximum (FWHM).

placed in the craniotomy and sealed with Vetbond (3M) and then secured with dental cement (C\&B Metabond). A $5 \mathrm{mg}$ per $\mathrm{kg}$ dose of carprofen was subcutaneously administered before awakening and for three additional days for analgesia. For head stabilization, a custom metal plate with a $6 \mathrm{~mm}$ central hole was attached to the skull. The headbar was secured very close to the skull to enable deep 3P imaging without steric hinderance of the objective. Custom 3D-printed headbar holders (CU Anschutz Optogenetics and Neural Engineering Core) were attached to a $200 \mathrm{~mm}$ breadboard (Thorlabs) affixed to stacked dual-axis goniometers (Optosigma, $+/-15^{\circ}$, $\mathrm{GOH}-65 \mathrm{~B} 76 \mathrm{R})$ such that the rotation center height was located at the headbar. Prior to imaging, the angle of the cranial window was adjusted to be perpendicular to the axis of the excitation beam using the goniometers. In vivo imaging measurements were taken either immediately following the surgery (acute preparation) or 2-3 weeks post-surgery (chronic preparation) as noted in the figures. During imaging sessions, mice were anesthetized with isoflurane and immobilized by attaching the head plate to the custom stage and temperature was monitored continuously as above. Images were acquired using Slidebook 2021 (3i) imaging software with 3 key modifications to standard laser point scanning systems for 3P imaging: 1) the laser was blanked during the galvanometer overscan to reduce the average power at the sample outside of the imaging FOV, 2) Slidebook 2021 was modified to allow for fine power control of the motorized half wave plate $(+/-0.1 \%)$, and 3 ) scanning was intermittently paused for 1 minute after every 3 minutes of continuous scanning to allow for heat dissipation ${ }^{20}$. Large $3 D$ structural $z$ stacks were acquired at $512 \times 512$ pixels, 2 $\mu$ s dwell time, and frame averaging $=2$, with a FOV of 385 x $385 \mu \mathrm{m}$ with a $3 \mu \mathrm{m}$ z-step. Z-stacks for optical measurements were acquired with the same settings without frame averaging and with a $0.5 \mu \mathrm{m}$ z-step. The power after the objective was measured daily by centering the galvanometers and acquiring a point-scan power curve on a high-power microscope slide meter sensor head (S175C, Thorlabs) in immersion water. The maximum power at the sample was 1-55 $\mathrm{mW}(0-1100 \mu \mathrm{m}$ depth).

\subsection{Image Processing and Analysis}

Image stacks were registered with StackReg (http://bigwww.epfl.ch/thevenaz/stackreg/) prior to analysis with Image $\mathrm{J}^{21}$. All images shown are either raw or filtered with a $0.5 \mu \mathrm{m}$ median filter, as noted in the figure legends. Z-width of max projected images are also noted in the figures. Signal to Background Ratio (SBR) analysis was performed using ImageJ on 3-10 cells located \pm 5 microns from the indicated depth. For each wavelength and pulse energy measured, a z-stack was 
acquired, and an ROI was drawn over the cell body of interest, and the Plot Z-Axis Profile ImageJ command was used to find the z-plane of peak intensity. All measurements were made at this single plane image. For the peak signal, a line scan was made through the cell body and the five pixel values surrounding the maximum were averaged. For SBR measurements of mScarlet, the background fluorescence was measured within the area of an unlabeled neuron to avoid measuring virally labeled neurites as background fluorescence. Plotting and statistical analyses were performed in JMP 15 (SAS). SBR measurements were expressed as Mean (SD). Logarithmic pulse energy vs. signal plots (Fig. 3c, Figs. S3-4) were analyzed using linear regression (MSE, mean square error) and the slopes were expressed as Mean \pm SEM. The pulse energy at the focal plane (z-depth) in Figs. S2, 4 was calculated with the equation below, using a linear extrapolation of previously published experimental effective attenuation lengths (EAL) in the mouse neocortex ${ }^{14,19,22}$, where $P=$ average power, $f=$ laser repetition rate $(1 \mathrm{MHz})$, and $\mathrm{z}=$ cortical depth:

$$
(P / f)_{\text {focal plane }}=(P / f)_{\text {surface }} * e^{-(z / E A L)}
$$

\section{Results:}

\subsection{Frequency-resolved optical gating pulse measurements}

To overcome the fundamental depth limit of $2 \mathrm{P}$ imaging in the mouse brain $^{23}$, we built a custom 3-photon microscope with a multichannel emission detection path (Fig. 1a). With the ultimate goal of measuring the in vivo excitation properties of RFPs for simultaneous dual-color 3P microscopy, we first characterized our output pulses after the objective at a range of wavelengths using frequency resolved optical gating (FROG $)^{27}$. Pulse characterization with FROG allows for the recovery of spectral intensity and phase using iterative spectrogram inversion algorithms and provides a ground-truth pulse width measurement ${ }^{25,26}$. We optimized the prism pulse compressor at $1300 \mathrm{~nm}$ to achieve $51 \mathrm{fs}$ pulses and a pulse duration of $51-75 \mathrm{fs}$ for 1225 to $1360 \mathrm{~nm}$, except for $1250 \mathrm{~nm}$, which was 104 fs (Fig. 1b). The raw spectrograms, retrieved spectral intensities, and phases are presented together in Figure S1. The prism compressor was optimized at $1300 \mathrm{~nm}$ and maintained at the same settings for all measurements.

\subsection{Nonlinear excitation properties of tdTomato in the living mouse brain at depth}

We implanted chronic cranial windows over the posterior parietal cortex (PPC) of triple transgenic mice (Olig2CreER;RCL-tdTomato;MOBP-EGFP,OTM) that express tdTomato in oligodendrocyte lineage cells and EGFP specifically in mature oligodendrocytes and myelin sheaths ${ }^{11}$. Single-excitation structural 3P microscopy of the brain volumes from OTM mice revealed single- and dual-labeled oligodendrocyte lineage cells throughout the cortical layers and subcortical white matter (Fig. 2a-b). To maintain constant average power at the brain surface, the laser power after the objective was measured at each wavelength and adjusted prior to imaging. We found that the pulse energies required for imaging were similar to those recently published for structural and functional neuronal imaging in vivo, and importantly, below the limits for heating- and nonlinear absorption-induced damage ${ }^{14,16,27}$. To determine the in vivo excitation properties of tdTomato, we measured the fluorescent signal, background, and signal-to-background ratio (SBR) in layer $6 b$ of the PPC (760 $\mu \mathrm{m}$ depth) of tdTomato at a range of wavelengths $(1225-1360 \mathrm{~nm})$ and constant average power at the surface (Fig. $\mathbf{2 c - g}$ ). The peak signal increased at 1300 vs. 1225 and $1250 \mathrm{~nm}$ (mean (SD) = 1606.98 (499.02) vs. 879.66 (260.38), 386.79 (110.631), respectively), and plateaued through $1360 \mathrm{~nm}$ (Fig. 2e), consistent with a broad excitation range for tdTomato in the $1300 \mathrm{~nm}$ range. However, we found that the background decreased from 1300 to $1360 \mathrm{~nm}$, resulting in an increased SBR in the $1325-1360 \mathrm{~nm}$ range when compared to $1300 \mathrm{~nm}$ (Fig. 2g, 21.91 (6.01), 22.39 (4.32), 30.74 (9.78) vs. 17.13 (5.32), respectively). To assess the contribution of $2 \mathrm{P}$ vs. $3 \mathrm{P}$ excitation processes to signal generation for each wavelength, we generated logarithmic signal vs. pulse energy plots at each wavelength for a range of pulse energies at the focal plane $(2.3-3.8 \mathrm{~nJ}$, Fig. S2). The slope of the logarithmic plot for tdTomato at $1225 \mathrm{~nm}$ was $2.171 \pm 0.526$, indicating a larger contribution of $2 \mathrm{P}$ than $3 \mathrm{P}$ excitation to the fluorescent emission at this wavelength and imaging depth. The log-plot slopes at 1300 and $1325 \mathrm{~nm}$ were $3.066 \pm 0.390$ and $2.874 \pm 0.369$, respectively, indicating that $3 \mathrm{P}$ excitation dominates in the $1300 \mathrm{~nm}$ range for tdTomato. These in vivo results are consistent with recently published $2 \mathrm{P}$ and $3 \mathrm{P}$ action cross sections of purified samples ${ }^{17}$. Our data indicate that the significant decrease in out-of-focus (2P) background with longerwavelength $3 \mathrm{P}$ excitation of tdTomato drives the observed increase in the SBR of this fluorophore.

\subsection{Nonlinear excitation properties of mScarlet in the living mouse brain at depth}

Historically, limitations in the maturation and quantum yield of monomeric RFPs have hampered their usage. Recently, a truly monomeric protein with high brightness and quantum yield, mScarlet, was developed ${ }^{28}$. This fluorophore has been included in a novel suite of viral tools used to express the highly sensitive, red-shifted opsin, ChRmine, to provide a red-shifted fluorescent reporter for structural imaging of targeted cells and subcellular structures ${ }^{18}$. To investigate the utility of mScarlet for dual color 3P microscopy, we injected AAV8hSyn-mScarlet-WPRE into the deep layers of the primary motor cortex to label motor output neurons. These injections were performed in MOBP-EGFP mice, which express EGFP in myelinating oligodendrocytes, to allow visualization of neuron-glia interactions. Single-excitation structural 3P microscopy of mScarlet-injected MOBPEGFP mice permitted the simultaneous visualization of excitatory neurons and mature oligodendrocytes at depths up to $1100 \mu \mathrm{m}$ in forelimb motor cortex (Fig. 3a). 
bioRxiv preprint doi: https://doi.org/10.1101/2021.10.18.464840; this version posted October 19, 2021. The copyright holder for this preprint (which was not certified by peer review) is the author/funder. All rights reserved. No reuse allowed without permission.
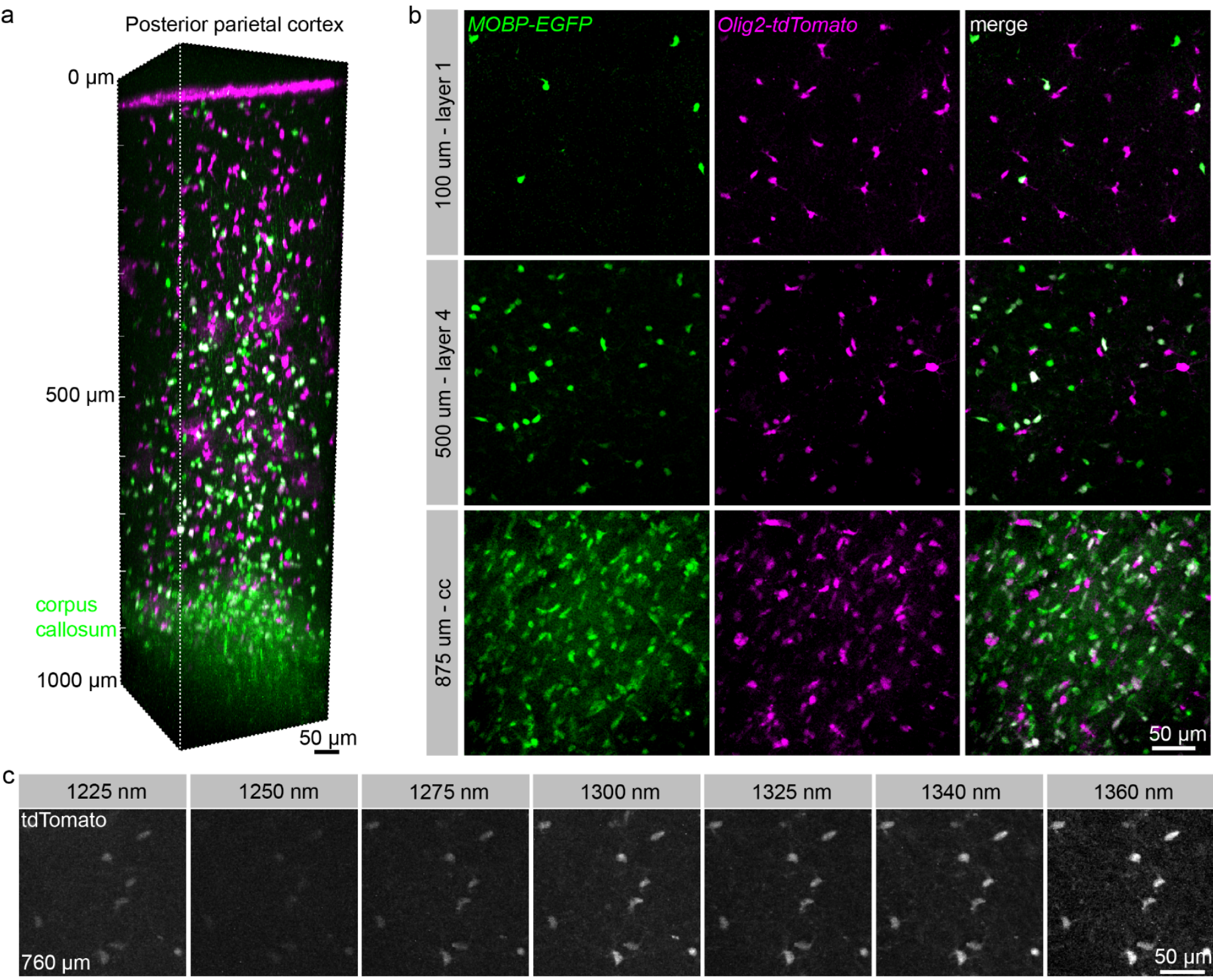
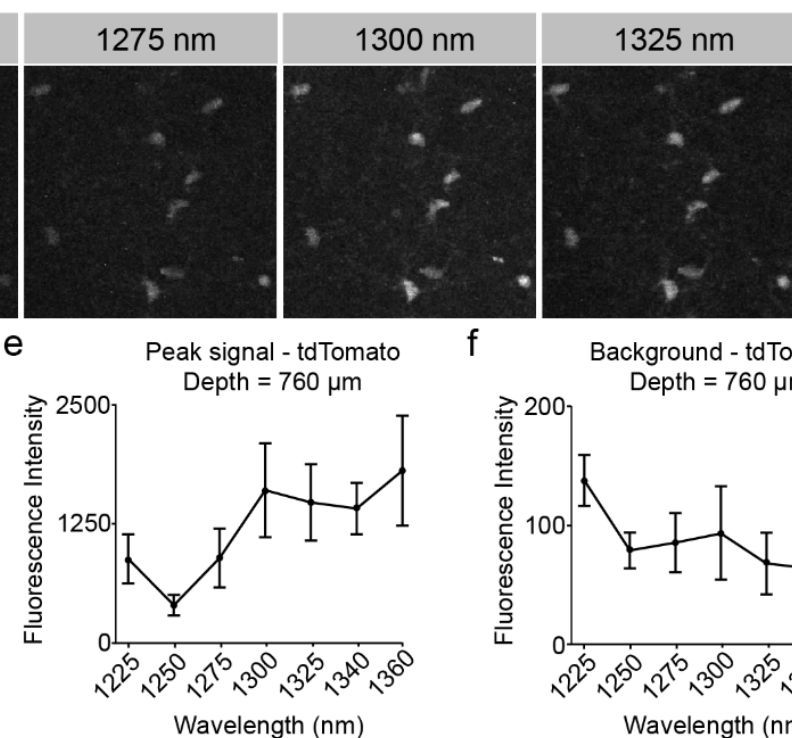



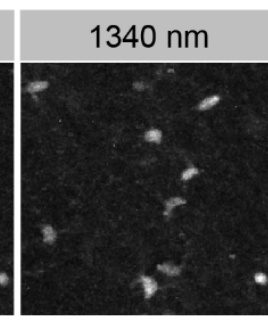

g

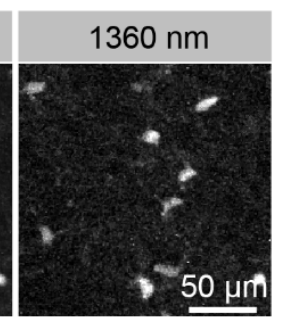

SBR - tdTomato Depth $=760 \mu \mathrm{m}$



Distance $(\mu \mathrm{m})$

Figure 2: Simultaneous 3P excitation of EGFP and tdTomato in posterior parietal cortex (PPC). a) 3D image volume from a chronic implanted cranial window in an Olig2-CreER; RCL-tdTomato; MOBP-EGFP mouse at P60. b) Max projections of $\sim 50 \mu \mathrm{m}$ volumes in cortical layers 1 (top), 4 (middle), and the corpus callosum (cc, bottom). Note the highly myelinated corpus callosum shows an increased density of dual-labeled mature oligodendrocyte cell bodies and strong MOBP-EGFP myelin signal compared to superficial cortex. c) Max projection images of $9 \mu \mathrm{m}$ volumes at $760 \mu \mathrm{m}$ depth and at a range of excitation wavelengths with average power $=45.2 \mathrm{~mW}$. d) Example line scan plots through a single tdTomato-positive oligodendrocyte cell body at 1225 and $1340 \mathrm{~nm}$ show increased signal and decreased background with longer wavelength excitation. e) Max fluorescence signal of tdTomato-positive oligodendrocyte lineage cells. f) Background fluorescence in the tdTomato detection channel. g) Signal to background ratio vs. wavelength comparison of the tdTomato signal at $760 \mu \mathrm{m}$ depth $(\mathbf{e}-\mathbf{g})$ Plots include measurements taken with average power $=45.2$ $\mathrm{mW}$ after the objective and pulse energy $=\sim 2.5-3.6 \mathrm{~nJ}$ at the focus. Data represent $\mathrm{n}=10$ cells from a triple transgenic mouse and are represented as the mean \pm standard deviation. Due to low output power at $1360 \mathrm{~nm}$, the data at this wavelength were acquired using $36 \mathrm{~mW}$ after the obiective and then scaled bv a value of $1.98=(45.2 / 36.0)^{3}$. 
bioRxiv preprint doi: https://doi.org/10.1101/2021.10.18.464840; this version posted October 19, 2021. The copyright holder for this preprint

(which was not certified by peer review) is the author/funder. All rights reserved. No reuse allowed without permission.



\section{b}
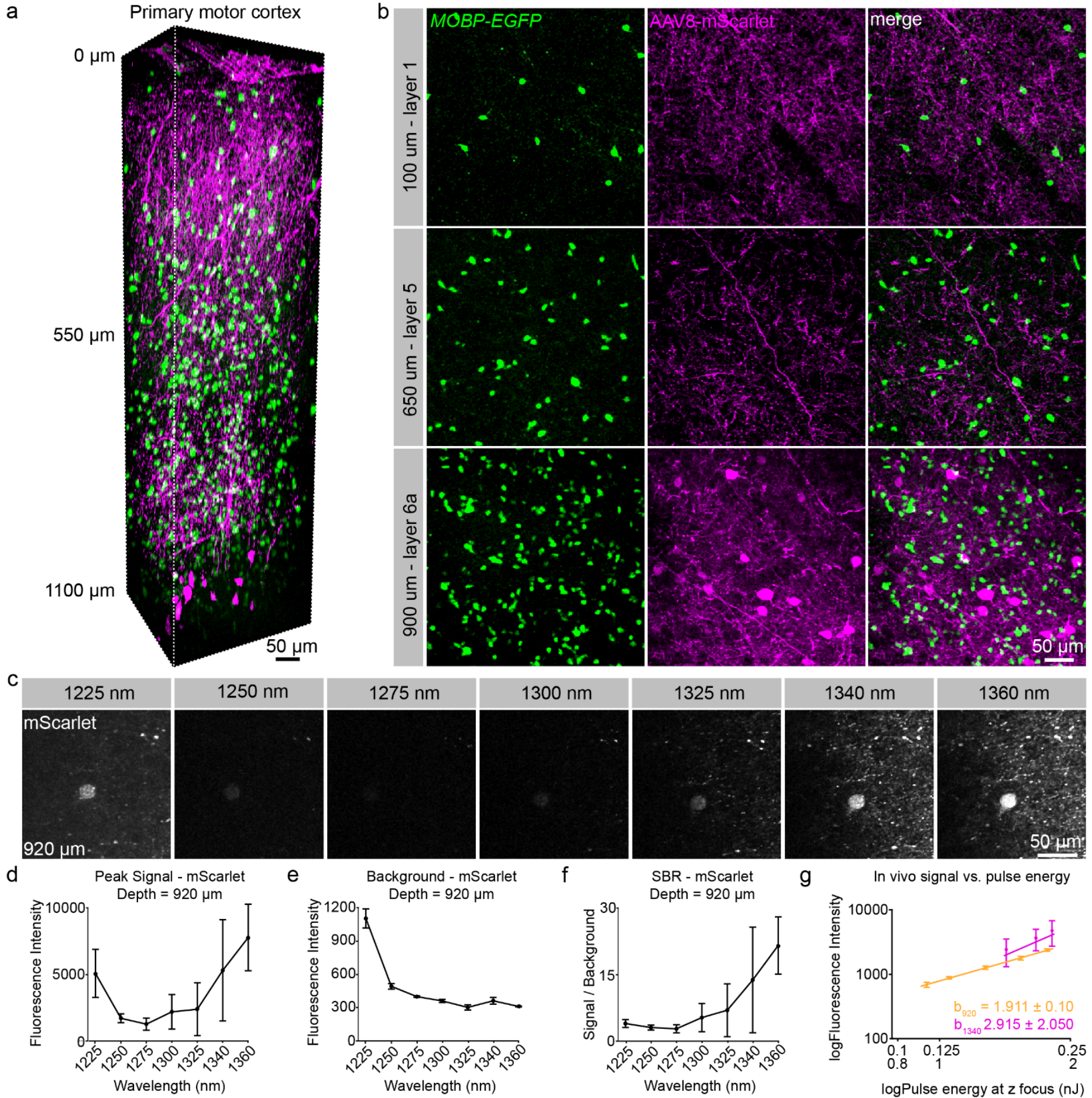

g

In vivo signal vs. pulse energy

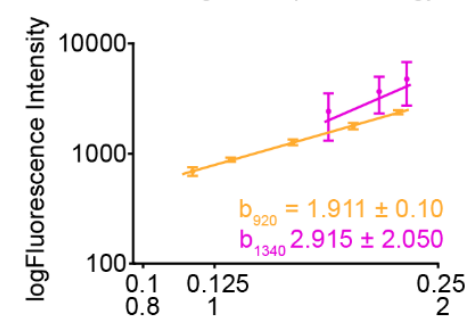

$\log P u l s e$ energy at $z$ focus $(n J)$

Figure 3: Simultaneous 3P excitation of EGFP and mScarlet in primary motor cortex. a) 3D image volume from an acutely implanted cranial window in an MOBP-EGFP mouse at P65 that was injected with AAV8-hsyn-mScarlet virus at 1000 and $750 \mu \mathrm{m}$ depths in the primary motor cortex. Note large mScarlet-positive layer $5 / 6$ motor output neurons are labeled at the bottom of the image volume and neuronal processes of these cells are labeled throughout the volume. b) Max projection images of $\sim 30 \mu \mathrm{m}$ volumes in cortical layers 1 (top), 5 (middle), and 6a (bottom). c) Max projection images of $9 \mu \mathrm{m}$ z-width at $920 \mu \mathrm{m}$ depth show differences in signal and background fluorescence across a range of excitation wavelengths. Note the increased signal and background at $1225 \mathrm{~nm}$. d) Max fluorescence signal of mScarlet-positive neurons for the range of wavelengths. e) Background fluorescence measurements in the mScarlet channel. f) Signal to background ratio vs. wavelength comparison of mScarlet signal at a depth of $920 \mu \mathrm{m}$ from the brain surface. g) log-log signal vs. pulse energy plots illustrate the contribution of 2- and 3P excitation to the fluorescence intensity for mScarlet at 920 (orange) and $1340 \mathrm{~nm}$ (magenta). The steeper slope of the $1340 \mathrm{~nm}$ line plots indicates a greater contribution of 3P excitation to the signal at the longer wavelength (theoretical $3 \mathrm{P} / 2 \mathrm{P}$ ratio $=1.5$ ). $\mathbf{d}-\mathbf{g}$ ) plots include measurements taken with average power $=47.7 \mathrm{~mW}$ after the objective and pulse energy $=\sim 1.3-2 \mathrm{~nJ}$ at the focus. Data represent $\mathrm{n}=3$ labeled neurons from a virally injected transgenic mouse and are represented as the mean \pm standard deviation. Due to low output power at $1360 \mathrm{~nm}$, the data at this wavelength were acquired using $38.5 \mathrm{~mW}$ after the objective and then scaled by a value of $1.90=(47.7 / 38.5)^{3}$. 
Large diameter layer 5 / 6 neuronal cell bodies were labeled at depths greater than $800 \mu \mathrm{m}$ and mScarletpositive processes were labeled throughout the cortical volume (Fig. 3a-b). To characterize the $2 \mathrm{P}$ excitation profile of mScarlet, we measured the in vivo $2 \mathrm{P}$ SBR of mScarlet at a range of wavelengths centered at $950 \mathrm{~nm}$ at a depth of $400 \mu \mathrm{m}$ from the brain surface. We found that the in vivo SBR of the two fluorophores intersected at $\sim 960 \mathrm{~nm}$ suggesting that this may be an ideal wavelength for 2P dual color imaging of EGFP and mScarlet (Fig. S3). However, we found acceptable dual-color cellular signal was achieved from $920-1000 \mathrm{~nm}$, highlighting the broad applicability of this fluorophore combination at wavelengths with sufficient output power from standard multiphoton lasers. Next, we measured the 3P SBR of $\mathrm{mScarlet}$ in the deep primary motor cortex $(920 \mu \mathrm{m}$ below the brain surface) at a range of excitation wavelengths and constant average power at the surface. We found that mScarlet showed a large signal increase at $1225 \mathrm{~nm}$ yet had a high out-of-focus background that substantially reduced the SBR at this wavelength (Fig. 3c-f), likely due to the predominance of $2 \mathrm{P}$ over $3 \mathrm{P}$ excitation at shorter wavelengths. While the $\mathrm{mScarlet}$ signal initially decreased from $1250-1300$, it subsequently increased from 1325 to $1360 \mathrm{~nm}$ (Fig. 3d). Unexpectedly, we found that the mScarlet background plateaued from $1300-1360$ $\mathrm{nm}$, despite choosing ROls within unlabeled neuronal shadows. However, these in vivo measurements may still overestimate the background at longer wavelengths due to strong diffuse labeling in the AAV-injected mice (Fig. 3c, e). The mean mScarlet SBR plateaued from $1340-$ $1360 \mathrm{~nm}$, which may represent an excitation peak for higher order excitation in the $1300 \mathrm{~nm}$ range (Fig. 3f). To determine the contributions of $2 \mathrm{P}$ versus $3 \mathrm{P}$ excitation we generated logarithmic signal vs. pulse energy plots at each wavelength for mScarlet as above (Fig. S4). We found that the slope of the logarithmic mScarlet plot at $1225 \mathrm{~nm}$ was $1.885 \pm 0.896$, reflecting the strong contribution of $2 \mathrm{P}$ excitation at this wavelength (Fig. S4, Fig. 3c-d). In contrast, we found that the slope at 1340 $\mathrm{nm}$ was 2.915 \pm 2.05 , confirming the predominant contribution of $3 \mathrm{P}$ excitation at wavelengths greater than $1325 \mathrm{~nm}$ for mScarlet (Fig. S4). Together, our data suggest that $\mathrm{mScarlet}$ is an attractive RFP with enhanced $3 \mathrm{P}$ cross section in the $1300 \mathrm{~nm}$ range for use in single excitation source dual color in vivo $3 \mathrm{P}$ imaging experiments.

\section{Discussion:}

4.1. tdTomato and mScarlet have enhanced threephoton cross sections in the $1250-1350 \mathrm{~nm}$ range

A recent study showed that while excitation in the 1600 $1700 \mathrm{~nm}$ range resulted in appreciable $3 \mathrm{P}$ excitation to the lowest energy state, certain RFPs have an order of magnitude larger 3P action cross section in the $1260-$ $1360 \mathrm{~nm}$ range ${ }^{17}$. Here, we took advantage of these $3 \mathrm{P}$ excitation properties to characterize the in vivo behavior of a classical tandem dimer RFP (tdTomato), as well as a recently developed monomeric RFP (mScarlet), for use in simultaneous dual-color 3-photon imaging studies. We found that both tdTomato and mScarlet have favorable 3photon excitation properties in the $1300-1340 \mathrm{~nm}$ range and we empirically defined the optimal excitation wavelengths for the best SBR at depth, which will be applicable to future dual-color in vivo imaging experiments. Similar to Hontani and colleagues ${ }^{17}$, we found that tdTomato exhibited a broad 3P excitation curve that increases significantly from $1250-1300 \mathrm{~nm}$ and plateaus through $1360 \mathrm{~nm}$. We found that the improvement in the signal to background ratio at the longer wavelengths $(1320-1360 \mathrm{~nm})$ was largely due to decreased out-of-focus background generation at these wavelengths. In contrast, the signal vs. wavelength curve for mScarlet was slightly right-shifted, as the peak signal increased significantly from $1325-1360 \mathrm{~nm}$ wavelengths, however, mScarlet also showed exceptional signal to background in this range. These results suggest that both of these red fluorophores have advantageous excitation properties in the short 3P wavelength range $(1300-1340$ $\mathrm{nm})$. Future characterization of additional red fluorophores in the $1300-1700 \mathrm{~nm}$ range will be useful to direct the application of both structural and functional in vivo dual-color $3 \mathrm{P}$ imaging.

\subsection{0 - $1360 \mathrm{~nm}$ excitation of RFPs likely represents excitation to a higher energy molecular state}

Certain red fluorescent proteins like Texas Red, tdTomato, and mScarlet exhibit short-wavelength onephoton absorption bands $(\sim 430-450 \mathrm{~nm})$ that represent excitation to higher-energy electronic states. Multiphoton excitation wavelengths used with classically employed fluorescent indicators likely induce excitation to the lowest-energy excited state (absorption peak), which has empirically generated optimal signal to noise in $2 \mathrm{P}$ experiments (e.g., $1040 \mathrm{~nm}$ excitation of tdTomato). However, our current findings suggest that $3 \mathrm{P}$ excitation is enhanced in these lower-wavelength absorption bands and has considerable implications for the design and implementation of future dual-color $3 \mathrm{P}$ studies. In addition, the $\sim 1260-1340 \mathrm{~nm}$ range of $3 \mathrm{P}$ excitation wavelengths overlaps with the long-wavelength tail of the $2 \mathrm{P}$ absorption cross section for most RFPs, therefore, signal detected at these wavelengths represents a mix of $2 \mathrm{P}$ and $3 \mathrm{P}$ excitation. We found that the mScarlet signal detected at $1225 \mathrm{~nm}$ was greater than in the $1250-1325$ range, likely due to the low energy tail in the mScarlet $2 \mathrm{P}$ absorption cross section curve. However, consistent with $2 \mathrm{P}$ excitation dominating at this wavelength, we measured high out-of-focus background generation at $1225 \mathrm{~nm}$ that severely decreases contrast and SBR at increased depths.

\subsection{Complete characterization of laser pulses for three-photon excitation in the 1200-1360 nm wavelength range}

We used frequency resolved optical gating (FROG) to measure the electric field of our laser pulses at the different wavelengths used for $3 \mathrm{P}$ microscopy. Previously, autocorrelation measurements in the $1200-$ 
$1400 \mathrm{~nm}$ range have been presented ${ }^{12,17}$. FROG measurements have an advantage over autocorrelation in that both the spectral intensity and phase of the electric field can be retrieved from the spectrograms and is useful for pulses with complex temporal profiles ${ }^{25}$. The retrieval of the electric field is done without prior assumptions of pulse shape, which are required for estimating pulse widths from autocorrelation measurements. Calculation of time-bandwidth product and the spectral phase can determine whether the pulse is optimally temporally compressed at the sample. While there are a variety of FROG instrument geometeries ${ }^{24}$, we used second harmonic generation (SHG)-FROG. One limitation of SHG-FROG is that the direction of time of the pulse is not captured in the measurement ${ }^{29}$. This makes timereversed traces in Fig. 1 equally likely and leads to ambiguity in the sign of the spectral phase. We measured an asymmetric pulse profile for 1250 and $1275 \mathrm{~nm}$ center wavelengths when tuning the output from the NOPA laser. Full characterization and correction for dispersion to allow the shortest pulse at the sample is especially important for 3P microscopy where the fluorescent signal, Is, is highly dependent on the pulse duration, $\tau$, by the relation, $I_{s} \sim \frac{1}{\tau^{2}} 32$.

In our characterization of the wavelength dependence of $3 \mathrm{P}$ imaging, we do expect a reduction of the overall fluorescent signal at $1250 \mathrm{~nm}$ and $1275 \mathrm{~nm}$ wavelengths due to the longer pulse durations. In addition, when there is a mixture of $2 \mathrm{P}$ and $3 \mathrm{P}$ excitation, as in the case of red fluorophores, we can approximate the SBR as:

$$
S B R_{\text {Total }} \sim S B R_{2 P}\left(1+\frac{S_{3 P}}{S_{2 P}}\right)
$$

Thus, the enhancement in SBR over $\mathrm{SBR}_{2 \mathrm{P}}$ depends on the ratio of the $3 \mathrm{P}$ signal versus $2 \mathrm{P}^{17}$. This ratio is affected by both the pulse duration, $\tau$, and the $2^{\text {nd }}$ and $3^{\text {rd }}$ order temporal coherence of the pulse $\left(g_{p}^{(2)} \text { and } g_{p}^{(3)}\right)^{17}$ in the form:

$$
\frac{S_{3 P}}{S_{2 P}} \sim \frac{g_{p}^{(3)}}{g_{p}^{(2)} \tau}
$$

Taking this into consideration, we also expect a reduction in the signal to background ratio (SBR) at 1250 and 1275 $\mathrm{nm}$ due to the longer pulse duration and asymmetric pulse shape.

\subsection{Advantages of single wavelength dual-color $3 P$ imaging}

Optical engineering of microscopes capable of simultaneous 1300 and $1700 \mathrm{~nm}$ 3P excitation presents multiple difficulties. Current commercially available optical elements (e.g., Pockels cells) are not commonly tested in the $1700 \mathrm{~nm}$ range and achieving adequate transmission of the excitation light out the objective may require additional purchases and/or custom modifications.
Additionally, $1700 \mathrm{~nm}$ light requires independent GDD optimization, potentially with a second prism compressor setup, and for simultaneous imaging, multiple excitation sources must be mixed and aligned for each imaging session. The results presented here suggest that for certain applications of structural dual color 3P excitation, specific fluorophore combinations can be used to exploit the enhanced 3P cross sections of RFPs in the $1300 \mathrm{~nm}$ range without the need for a second $1700 \mathrm{~nm}$ excitation path. Still, the development of simultaneous 1300/1700 $\mathrm{nm}$ systems will be important for future studies using dynamic optochemical indicators and/or optical stimulation deep in the brain. For example, the large spectral separation between the 1300 and $1700 \mathrm{~nm}$ ranges could allow for dual-color stimulation and recording experiments with opsin/indicator combinations like ChRmine ${ }^{18}$ and GCaMP, without cross-excitation of the indicator by the stimulation wavelength. Furthermore, it was reported that unlike tdTomato and mScarlet, mCherry exhibits a 7-fold decreased 3P cross section in the $1300 \mathrm{~nm}$ range compared to $1650 \mathrm{~nm}^{17}$ and thus may require a $1300 / 1700 \mathrm{~nm}$ system to be optimally employed as a fluorescent reporter for dual-color $3 \mathrm{P}$ imaging.

\subsection{Scattering and absorption in the $1300-1400 \mathrm{~nm}$ range}

The effective attenuation length (EAL) for 3P microscopy is defined as the depth at which the unscattered excitation light is reduced by $1 / \mathrm{e}^{3}$. In the mouse brain, both $2 \mathrm{P}$ and 3P EAL are determined by a combination of scattering and water absorption. 2P and 3P EALs in different regions of the mouse brain have been characterized extensively, both in vivo and in silico ${ }^{12,14,19,20,27}$. Therefore, considerations such as the scattering and water absorption, as well as the output power from current NOPA configurations should be taken into account for dual-color $3 \mathrm{P}$ imaging with a single excitation source in the $1300 \mathrm{~nm}$ range. In this study, we did not achieve sufficient output power at $1 \mathrm{MHz}$ and wavelengths greater than $1340 \mathrm{~nm}$ to allow for practical deep in vivo imaging, however, excitation in the $1325-1340 \mathrm{~nm}$ range provided exceptional SBR for both tdTomato and mScarlet. Because water absorption increases exponentially between 1340 and $1400 \mathrm{~nm}$, our results are encouraging in that a narrow excitation window $(1320-1340 \mathrm{~nm})$ may be used to excite a broad range of green and red fluorescent reporter combinations with a single wavelength. Future work includes empirically testing a suite of fluorescent reporters for use in multicolor $3 \mathrm{P}$ experiments, using emission path engineering to increase the number of detection channels, and defining $3 \mathrm{P}$ activation properties of opsins and other dynamic indicators.

Acknowledgments: We thank Dr. Chris $X u$ for his invaluable advice in setting up the 3P microscope at the University of Colorado Anschutz Medical Campus; Dr. Karl Deisseroth for providing us with the AAV8-hSynmScarlet-WPRE virus; Anthony Chavez for technical and veterinary assistance; Andrew Scallon and the CU 
Anschutz Optogenetics and Neural Engineering Core (P30NS048154) for 3D printing and stage design; Dr. Michael Young for optics assistance; members of the Hughes and Welle labs for discussions.

Funding: M.A.T. is supported by National Institutes of Health NINDS (F31NS120540). Funding was provided by NINDS NS115975, University of Colorado Department of Cell and Developmental Biology Pilot Grant, the Whitehall Foundation, and the National Multiple Sclerosis Society (RG-1701-26733) to E.G.H. Funding was provided by NINDS (UF1 NS116241) and the National Science Foundation (BCS-1926676) to E.A.G. and D.R.

Contributions: E.G.H, E.A.G., and M.A.T. conceived the project. G.L.F and E.A.G. built the microscope. M.A.T. conducted experiments and generated Figs. 1a, 2, 3, S1a, S3, S4, and S5. G.L.F. designed and contributed Figs. 1b, S1b, and S2. M.E.S. analyzed data in Figs. 2, 3, S3, and S5. B.N.O. and K.K. developed the software and helped with microscope setup. E.G.H., E.A.G., and D.R. supervised all experiments. M.A.T. and E.G.H. wrote the manuscript with input and editing from all other authors.

Competing Interests: K.K. is a co-founder and part owner of $3 \mathrm{i}$. The other authors declare no competing financial interests.

Data and materials availability: All data that support the findings, tools, and reagents will be shared on an unrestricted basis; requests should be directed to the corresponding authors.

\section{List of Supplementary Materials:}

Supplementary Figures 1 to 4

\section{References:}

1. E. E. Hoover and J. A. Squier, "Advances in multiphoton microscopy technology," Nat. Photonics 7(2), 93-101 (2013) [doi:10.1038/nphoton.2012.361].

2. F. Helmchen and W. Denk, "Deep tissue two-photon microscopy," Nat. Methods 2(12), 932-940 (2005) [doi:10.1038/nmeth818].

3. T. Wang and C. Xu, "Three-photon neuronal imaging in deep mouse brain," Optica 7(8), $947 \quad$ (2020) [doi:10.1364/OPTICA.395825].

4. M. A. Gaffield et al., "Inhibition gates supralinear $\mathrm{Ca} 2+$ signaling in Purkinje cell dendrites during practiced movements," eLife 7, J. L. Raymond et al., Eds., e36246, eLife Sciences Publications, Ltd (2018) [doi:10.7554/eLife.36246].

5. S. Han, W. Yang, and R. Yuste, "Two-Color Volumetric Imaging of Neuronal Activity of Cortical Columns," Cell Rep. 27(7), 2229-2240.e4 (2019) [doi:10.1016/j.celrep.2019.04.075].

6. H. Kawano et al., "Two-photon dual-color imaging using fluorescent proteins," Nat. Methods 5(5), 373-374 (2008) [doi:10.1038/nmeth0508-373].
7. C. Xu and W. W. Webb, "Measurement of two-photon excitation cross sections of molecular fluorophores with data from 690 to $1050 \mathrm{~nm}$," J. Opt. Soc. Am. B 13(3), 481 (1996) [doi:10.1364/JOSAB.13.000481].

8. C. Ricard et al., "Two-photon probes for in vivo multicolor microscopy of the structure and signals of brain cells," Brain Struct. Funct. 223(7), 3011-3043 (2018) [doi:10.1007/s00429018-1678-1].

9. M. Drobizhev et al., "Two-photon absorption properties of fluorescent proteins," Nat. Methods 8(5), 393-399 (2011) [doi:10.1038/nmeth.1596].

10. H. Dana et al., "Sensitive red protein calcium indicators for imaging neural activity," eLife 5, M. Häusser, Ed., e12727, eLife Sciences Publications, Ltd (2016) [doi:10.7554/eLife.12727].

11. E. G. Hughes et al., "Myelin remodeling through experiencedependent oligodendrogenesis in the adult somatosensory cortex," Nat. Neurosci. 21(5), 696-706 (2018) [doi:10.1038/s41593-018-0121-5].

12. N. G. Horton et al., "In vivo three-photon microscopy of subcortical structures within an intact mouse brain," Nat. Photonics 7(3), 205-209

(2013) [doi:10.1038/nphoton.2012.336].

13. D. G. Ouzounov et al., "In vivo three-photon imaging of activity of GCaMP6-labeled neurons deep in intact mouse brain," Nat. Methods 14(4), 388-390 (2017) [doi:10.1038/nmeth.4183].

14. T. Wang et al., "Quantitative analysis of $1300-\mathrm{nm}$ threephoton calcium imaging in the mouse brain," eLife 9, R. Yasuda and C. Dulac, Eds., e53205, eLife Sciences Publications, Ltd (2020) [doi:10.7554/eLife.53205].

15. K. Guesmi et al., "Dual-color deep-tissue three-photon microscopy with a multiband infrared laser," Light Sci. Appl. 7(1), 12 (2018) [doi:10.1038/s41377-018-0012-2].

16. M. Yildirim et al., "Functional imaging of visual cortical layers and subplate in awake mice with optimized three-photon microscopy," Nat. Commun. 10(1), 177 (2019) [doi:10.1038/s41467-018-08179-6].

17. Y. Hontani, F. Xia, and C. Xu, "Multicolor three-photon fluorescence imaging with single-wavelength excitation deep in mouse brain," Sci. Adv. 7(12), eabf3531, American Association for the Advancement of Science (2021) [doi:10.1126/sciadv.abf3531].

18. J. H. Marshel et al., "Cortical layer-specific critical dynamics triggering perception," Science 365(6453), eaaw5202 (2019) [doi:10.1126/science.aaw5202].

19. C. M. Bacmeister et al., "Motor learning promotes remyelination via new and surviving oligodendrocytes," 7, Nat. Neurosci. 23(7), 819-831, Nature Publishing Group (2020) [doi:10.1038/s41593-020-0637-3].

20. K. Podgorski and G. Ranganathan, "Brain heating induced by near-infrared lasers during multiphoton microscopy," J. Neurophysiol. 116(3), 1012-1023, American Physiological Society (2016) [doi:10.1152/jn.00275.2016]. 
21. C. A. Schneider, W. S. Rasband, and K. W. Eliceiri, "NIH Image to ImageJ: 25 years of image analysis," Nat. Methods 9(7), 671-675 (2012) [doi:10.1038/nmeth.2089].

22. M. Wang et al., "Comparing the effective attenuation lengths for long wavelength in vivo imaging of the mouse brain," Biomed. Opt. Express 9(8), 3534-3543, Optical Society of America (2018) [doi:10.1364/BOE.9.003534].

23. P. Theer and W. Denk, "On the fundamental imaging-depth limit in two-photon microscopy," J. Opt. Soc. Am. A Opt. Image Sci. Vis. 23(12), 3139-3149 (2006) [doi:10.1364/josaa.23.003139].

24. R. Trebino et al., "Measuring ultrashort laser pulses in the time-frequency domain using frequency-resolved optical gating," Rev. Sci. Instrum. 68(9), 3277-3295, American Institute of Physics (1997) [doi:10.1063/1.1148286].

25. D. J. Kane, "Recent progress toward real-time measurement of ultrashort laser pulses," IEEE J. Quantum Electron. 35(4), 421-431 (1999) [doi:10.1109/3.753647].

26. D. M. Farinella et al., "Improving laser standards for threephoton microscopy," Neurophotonics 8(1), 015009, SPIE (2021) [doi:10.1117/1.NPh.8.1.015009].

27. C. J. Liu et al., "Three-photon imaging of synthetic dyes in deep layers of the neocortex," Sci. Rep. 10(1), 16351 (2020) [doi:10.1038/s41598-020-73438-w].

28. D. S. Bindels et al., "mScarlet: a bright monomeric red fluorescent protein for cellular imaging," Nat. Methods 14(1), 53-56 (2017) [doi:10.1038/nmeth.4074].
29. J. Paye et al., "Measurement of the amplitude and phase of ultrashort light pulses from spectrally resolved autocorrelation," Opt. Lett. 18(22), 1946-1948, Optical Society of America (1993) [doi:10.1364/OL.18.001946].

30. R. Trebino and D. J. Kane, "Using phase retrieval to measure the intensity and phase of ultrashort pulses: frequencyresolved optical gating," JOSA A 10(5), 1101-1111, Optical Society of America (1993) [doi:10.1364/JOSAA.10.001101].

31. M. Wang et al., "In vivo three-photon imaging of deep cerebellum," in Multiphoton Microscopy in the Biomedical Sciences XVIII 10498, pp. 122-127, SPIE (2018) [doi:10.1117/12.2285406].

32. Xu C et al., "Multiphoton fluorescence excitation: new spectral windows for biological nonlinear microscopy." P Natl Acad Sci Usa. 1996;93(20):10763-10768. doi:10.1073/pnas.93.20.10763. 


\section{SUPPLEMENTARY MATERIALS}

\section{Characterization of red fluorescent reporters for dual-color in vivo three-photon microscopy}

Michael A. Thornton ${ }^{1,2}$, Gregory L. Futia ${ }^{3}$, Michael E. Stockton ${ }^{1,2}$, Baris N. Ozbay ${ }^{4}$, Karl Kilborn ${ }^{4}$, Diego Restrepo ${ }^{1,2}$, Emily A. Gibson ${ }^{2,3,5}$, Ethan G. Hughes ${ }^{1,2,5}$ 

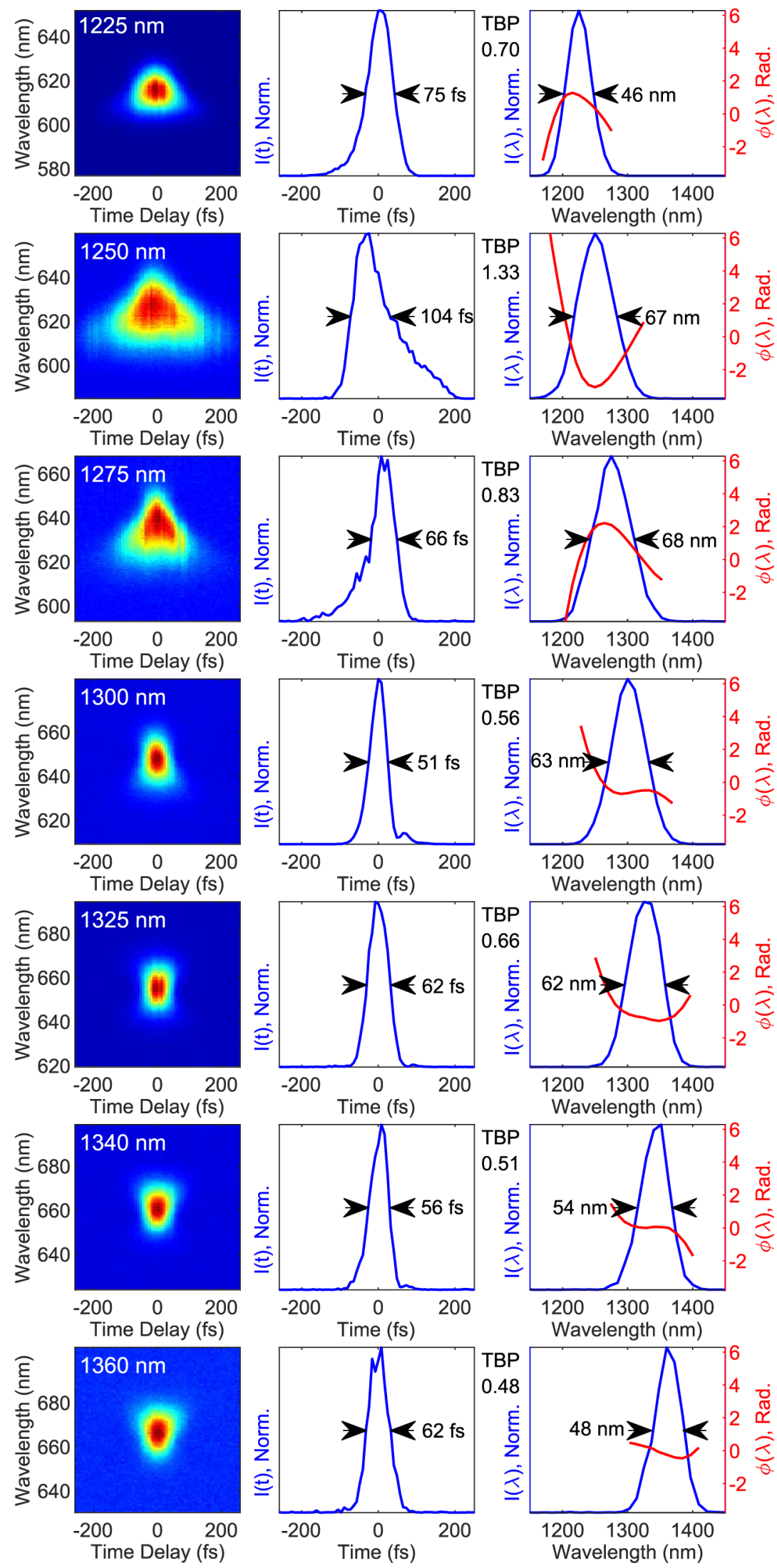

Supplementary Figure 1: Measured FROGscan spectrograms and retrieved temporal and spectral profiles. left) Measured FROGscan spectrograms at each investigated wavelength. middle) Retrieved temporal intensity I(t), as in Fig. 1. (right) Retrieved spectral intensity, $I(\lambda)$, with overlaid spectral phase, $\varphi(\lambda)$, in radians (red) with time-bandwidth product 
(TBP) annotated between the traces. The larger phases on the pulses at $1250 \& 1275 \mathrm{~nm}$ led to broader temporal pulses at these wavelengths and larger TBP. Log-Log Multiphoton Excitation Plots - tdTomato
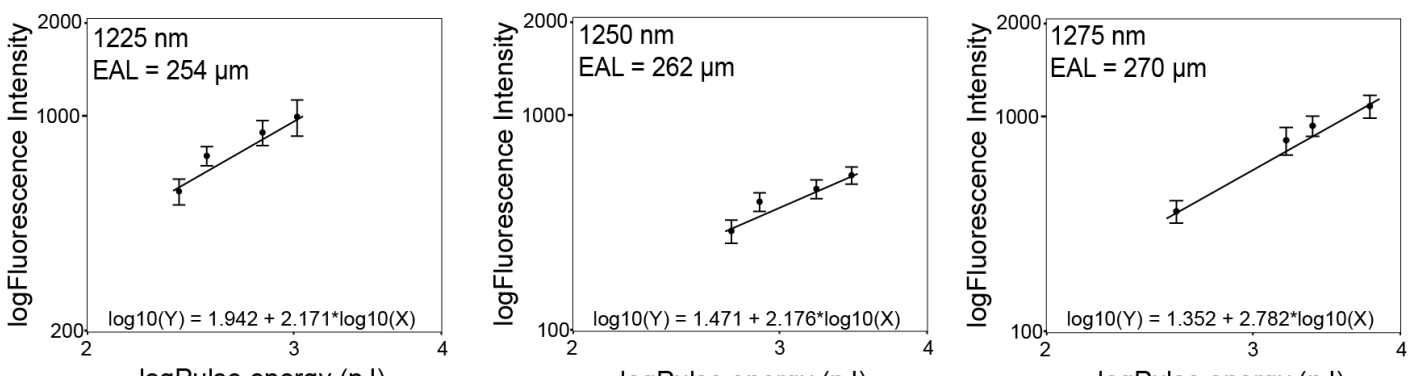

logPulse energy (nJ)
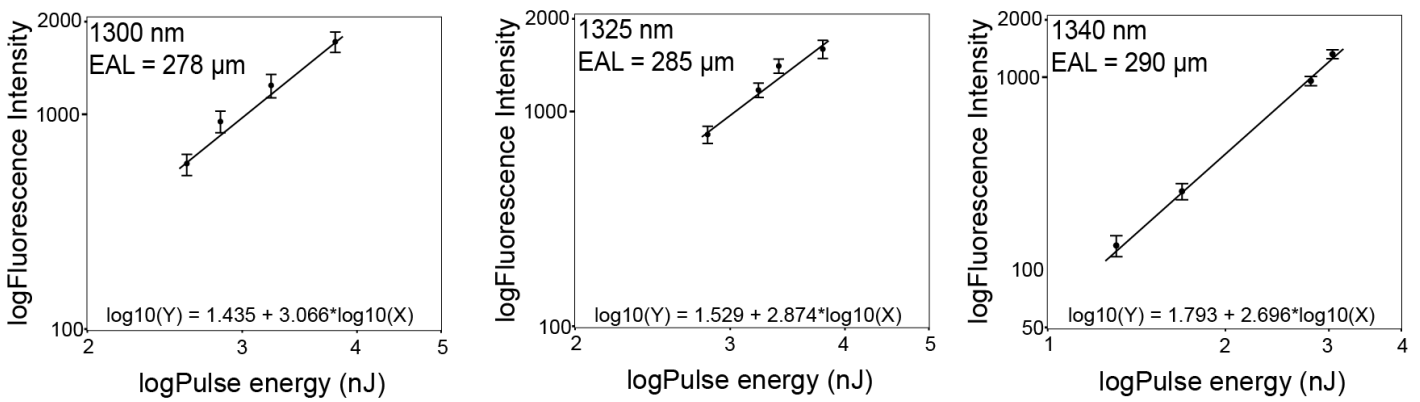

\begin{tabular}{|c|c|}
\hline Wavelength $(\mathrm{nm})$ & Slope \\
\hline 1225 & $2.171 \pm 0.526$ \\
\hline 1250 & $2.176 \pm 0.524$ \\
\hline 1275 & $2.782 \pm 0.382$ \\
\hline 1300 & $3.066 \pm 0.390$ \\
\hline 1325 & $2.874 \pm 0.369$ \\
\hline 1340 & $2.696 \pm 0.132$ \\
\hline
\end{tabular}

Supplementary Figure 2: Log-Log pulse energy vs. signal plots for tdTomato at $760 \mu \mathrm{m}$ below the brain surface. a) individual logarithmic plots of pulse energy vs. in vivo fluorescent signal at a range of 3P wavelengths. b) Table of slope values for the excitation power ramp lines shown in (a). The steeper slope at higher wavelengths indicates a greater contribution of $3 \mathrm{P}$ vs. $2 \mathrm{P}$ excitation. 

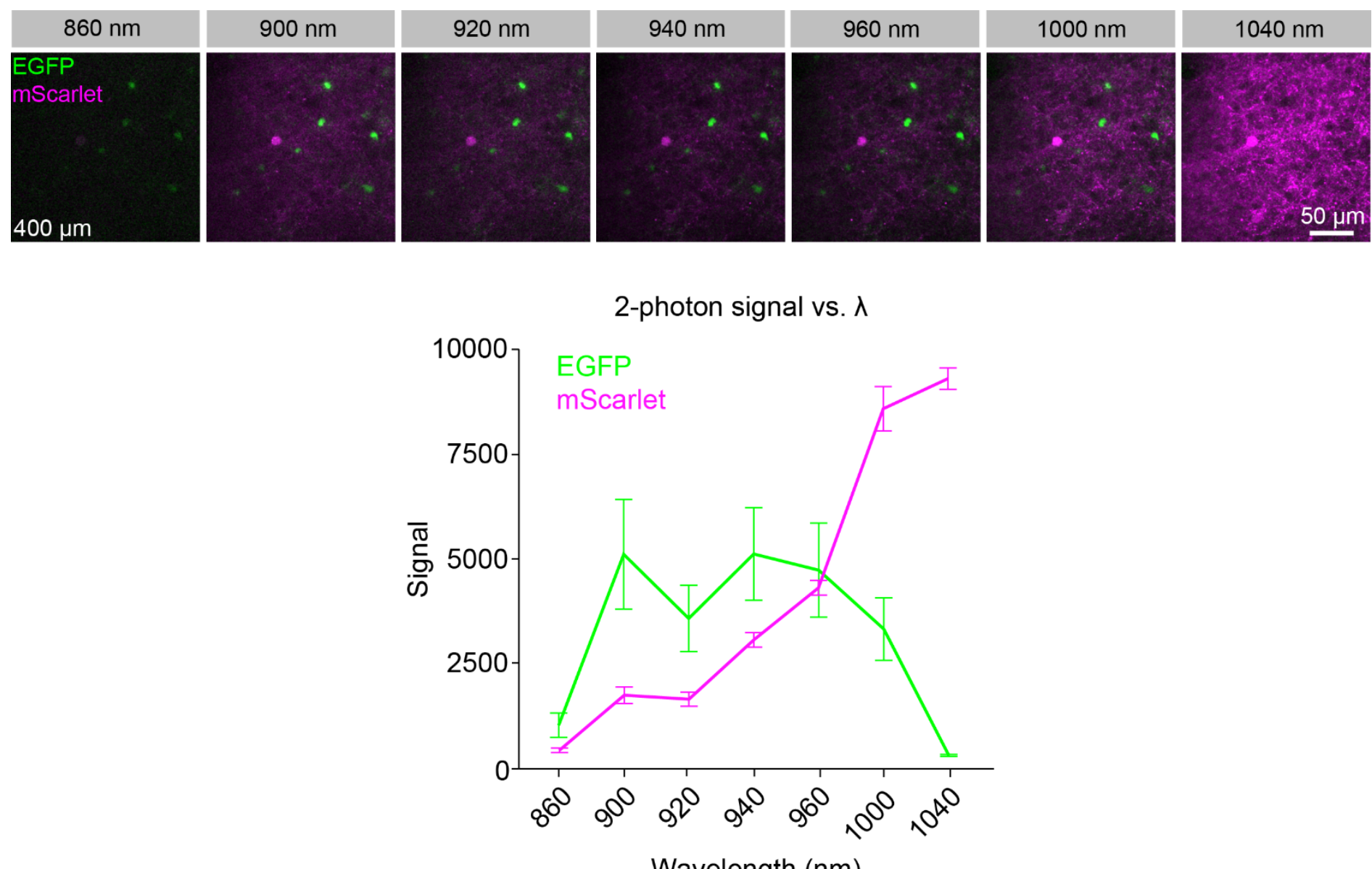

Supplementary Figure 3: Two-photon in vivo imaging of EGFP and mScarlet at a depth of $\mathbf{4 0 0} \boldsymbol{\mu m}$ from the pial surface. Single plane images from a chronic implanted cranial window in an AAV8-hSyn-mScarlet injected MOBP-EGFP mouse with varying excitation wavelength $(860-1040 \mathrm{~nm})$ and quantification of in vivo signal generation compared to excitation wavelength. The pulse energy at the focus was $.25 \mathrm{~nJ}$ at $920 \mathrm{~nm}$, the average power at the surface was kept constant, and the pulse width was adjusted to maximum signal at each wavelength. 

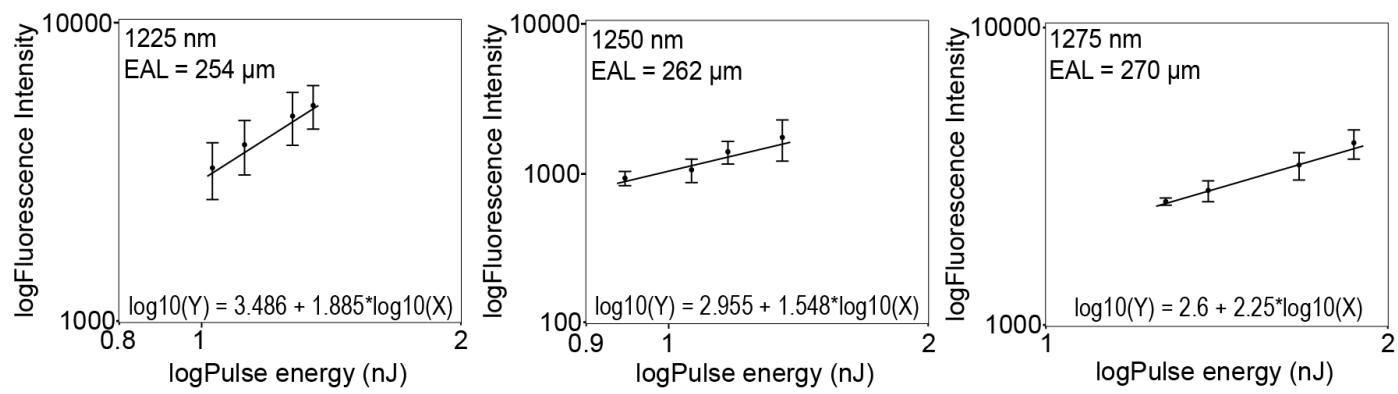

\begin{tabular}{|c|c|}
\hline Wavelength $(\mathrm{nm})$ & Slope \\
\hline 1225 & $1.885 \pm 0.896$ \\
\hline 1250 & $1.548 \pm 0.714$ \\
\hline 1275 & $2.250 \pm 0.552$ \\
\hline 1300 & $2.595 \pm 1.688$ \\
\hline 1325 & $2.496 \pm 2.209$ \\
\hline 1340 & $2.915 \pm 2.050$ \\
\hline
\end{tabular}
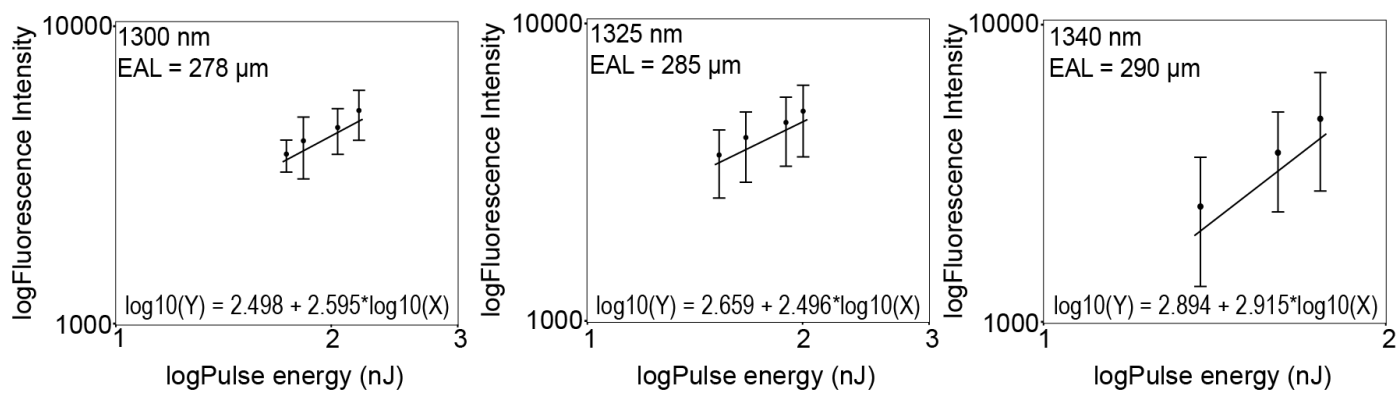

Supplementary Figure 4: Log-Log pulse energy vs. signal plots for mScarlet at $\mathbf{9 2 0} \boldsymbol{\mu m}$ below the brain surface. a) Individual logarithmic plots of pulse energy vs. in vivo fluorescent signal at a range of $3 \mathrm{P}$ wavelengths. b) Table of slope values for the excitation power ramp lines shown in (a). 